\section{NUCLEOTIDES}

\section{Flucluating Pools}

from our Cell Biology Correspondent

IN January (Nature, 229, 156; 1971) I drew attention to the elegant experiments of Nordenskjold and his colleagues who, exploiting their enzymatic assay for deoxynucleoside triphosphates, managed to measure the pool sizes of these molecules in mouse embryo cells. Skoog and Nordenskjold (Europ. J. Biochem., 19, 81; 1971) have now used their technique to measure changes in the four deoxynucleoside triphosphate pools during the inhibition and restoration of DNA synthesis following the addition and removal of hydroxyurea and cytosine arabinoside.

After inducing DNA synthesis in resting cultures of mouse embryo cells by increasing the concentration of serum in the medium from 0.5 per cent to 100 per cent, they assayed the pool sizes of dATP, dTTP, dGTP and dCTP as the cultures achieved maximal DNA synthesis. At their peaks, the pools of dATP and dTTP are in the range 3 to 4 pmoles per $\mu \mathrm{g}$ of DNA; the pool of $\mathrm{dCTP}$ is about 9 pmoles per $\mu \mathrm{g}$ of DNA whereas that of dGTP, the smallest of the four, is only 0.5 pmoles per $\mu \mathrm{g}$ DNA, which is sufficient to support DNA synthesis for only 30 seconds. When hydroxyurea $(1-10 \mathrm{mM})$ is added to these cells, DNA synthesis decreases rapidly and within a few minutes the inhibition is maximal. Measurements of the pool sizes of the four triphosphates during the establishment of this inhibition reveal an almost instantaneous loss of the dGTP pool and a more gradual decrease in the pool of dATP. The pools of the other two triphosphates dCTP and dTTP increase but the turnover of dTTP is reduced. Following removal of the inhibitor the pools are rapidly re-established at their normal levels but restoration of DNA synthesis lags behind. All these changes are consistent with the suggestion that hydroxyurea blocks the activity of ribonucleoside diphosphate reductase, and because of this, and the very small pool of dGTP, the drug causes very rapid inhibition of DNA synthesis.

Cytosine arabinoside inhibits DNA synthesis in mouse embryo cells at much lower concentrations (1-10 uM) than does hydroxyurea, and it causes very different changes in the sizes of the nucleotide pools. In the presence of cytosine arabinoside the pools of dATP, dGTP and dTTP doubled over a period of half an hour and continued to increase thereafter, albeit at a slower rate. By contrast, the pool of dCTP shrinks during the first hour and only slowly recovers its normal level. The dTTP pool turns over more slowly in cells treated with cytosine arabinoside than in controls (half-lives $13 \mathrm{~min}$ and 4 min respectively), but the pool is two to three times larger in the treated cells. Removal of the drug is rapidly followed by a restoration of the normal pool sizes and subsequently DNA synthesis restarts.

These findings rule out the idea that cytosine arabinoside blocks ribonucleoside diphosphate reductase activity; so how does it inhibit DNA synthesis? Cytosine arabinoside is triphosphorylated in mouse embryo cells and the parallels between the accumulation and loss of aCTP and the inhibition and restoration of DNA synthesis observed by Skoog and Nordenskjold strongly support the suggestion, first muted by Furth and Cohen in 1967, that aCTP, and therefore cytosine arabinoside, inhibits DNA synthesis by interfering with polymerization. Be that as it may, thanks to the valuable work of Nordenskjold and his colleagues there is now a much clearer picture of precisely what happens inside cells when they are exposed to these inhibitors.

\section{ORIENTATION}

\section{Jumping Spider}

from our Animal Behaviour Correspondent

IF a jumping spider (Salticidae) detects a moving object with its lateral eyes, it often turns so that the object can be identified by the principal eyes situated at the front of the head. Depending on the nature of the object, prey catching, mating or escape may then follow. The turn made by the spider is very accurate-it turns through just the right angle to bring the object into the field of the principal eyes.

How is this accuracy achieved? One possible mechanism is by visual feedback resulting from the spider's own movement, but M. F. Land (J. Exp. Biol., 54, 119; 1971) has shown that such a "closed-loop" system cannot be controlling the turns, because the spider turns correctly even when denied such feedback. Land anchored jumping spiders with a piece of card glued onto the back and gave them light card-

\title{
Geometries of Molecular Oxygen Complexes
}

IN next Monday's Nature Physical Science, Mingos presents a general treatment of factors affecting the geometry of dioxygen (molecular oxygen) complexes which might aid in resolving the problem of the geometry of oxygen binding in oxy-haemoglobin and oxymyoglobin. The basis of the argument is an approximate form of the secondorder Jahn-Teller effect. Whereas the first-order Jahn-Teller effect deals with distortions of geometry arising from degenerate electronic states, the second order effect is concerned with states which are merely close to each other (in the approximation used only the ground and first excited states are taken into consideration).

The effect provides a symmetry rule for predicting the stable shapes of molecules (R. G. Pearson, J. Amer. Chem. Soc., $91,4947 ; 1969)$ and suggests that, in the formation of complexes between Lewis acids and bases, it is only when the donor and acceptor orbitals do not have the same symmetry that distortion leading to a new equilibrium configuration of the complex will be allowed. In the particular case of dioxygen complexes, the distortion which would allow transformation of the $\pi$-bonded geometry M... I (which is energetically preferred if dioxygen acts as a Lewis base) into the non-linear configuration $\mathrm{M}-\mathrm{O}^{\mathrm{O}}$ is not expected. Peroxo-complexes and superoxo-complexes may be considered as Lewis acid complexes of dioxygen and will only have a non-linear geometry if the donating metal orbital is a $\sigma$-type orbital. The results are in agreement with observation for a range of mono and bi-nuclear dioxygen, superoxo-metal and peroxo-metal complexes.

For oxy-haemoglobin and oxy-myoglobin the treatment supports the $\pi$ bonded (Griffith) structures-Fe. . ?

The alternative formulation of these species as superoxo-complexes would not suggest distortion because the necessary electron transfer from metal to ligand orbitals does not involve the metal $\sigma$-orbitals. This result must, at present, be regarded with caution for two reasons: first, the treatment is based on an approximation, which has been widely successful but which Pearson considers can only be justified if it works in the application at hand; second, the argument takes no account of non-bonded repulsions between the oxygen atoms and the pyrrole nitrogen atoms of the porphyrin ring. Hoard (in Structural Chemistry and Molecular Biology (edit. by A. Rich and N. Davidson), W. H. Freeman, San Francisco, $573 ; 1968)$ has argued forcefully that the $\pi$-bonded geometry would involve considerable steric strain in haemoxygen complexes which could not readily be relieved. Mingos suggests that molybdenum (VI) and titanium (IV) $\pi$-peroxo complexes, perhaps with constrained ligands, would provide useful model systems for studying the influence of steric effects on metal-dioxygen complex geometries. 